\title{
Wind Variations of Wolf-Rayet Stars
}

\author{
Anthony F.J. Moffat \\ Département de physique, Université de Montréal, C.P. 6128, Succ. C-V, Montréal, \\ PQ, H3C 3J7, Canada, and Observatoire du mont Mégantic. Killam Research Fellow \\ of the Canada Council for the Arts.
}

\begin{abstract}
What distinguishes WR stars from most of their progenitor phases is their dense winds, that often completely hide their small hydrostatic cores. Thus, to learn about these cores, which are especially important in an evolutionary context, we are generally limited to studying the winds only. One way to do this is to examine their ubiquitous variability. Variability studies of (single) WR stars using various techniques have revealed two main classes of wind variation: (1) large-scale and periodic, probably related to rotating disturbances (e.g. NRP or magnetic structures) close to the core surface, and (2) small (multi-) scale and stochastic, probably intrinsic to the winds themselves. The former is only seen rarely in WR stars, contrary to other hot stars. The latter appears to be universal in WR and probably (though less obvious) in all hot-star winds.
\end{abstract}

\section{Types of Variability}

Wolf-Rayet (WR) stars have the strongest winds among stable massive stars. However, evidence accumulated over the past few years indicates that WR winds are far from steady and smooth, as was once assumed. This is deduced from the fact that (single) WR stars are generally quite variable, mostly in their strong emission lines. Since most WR winds are so dense that we cannot see their hydrostatic cores, this variability must be occuring in the winds themselves in most cases. By quantifying the nature of the variability, we can therefore explore not only properties of the winds, but also the origin of the variability, which may lie hidden from direct view.

Several techniques have been applied to reveal wind variabilty in WR stars (e.g. photometry, polarimetry, spectroscopy, or combinations thereof), but the most effective so far has been the study of optical line profile variations (LPVs). Previous work has often concentrated on UV LPVs, obtained using IUE (e.g. St-Louis 1990). However, the UV data are most effective in revealing LPVs in the $\mathrm{P}$ Cygni absorption profiles of mainly strong, often resonance, lines that prevail in the UV. This limits their visibility to the part of the wind that lies in the column towards the star. Despite the many interesting UV results, I will concentrate on the more global LPVs seen in emission lines in the optical, where it has been easier to get high-quality data. According to a recent study (Lépine \& Moffat 1999), optical wind LPVs can be sorted into three categories: 
1. Stochastic (S). All hot-star winds observed appropriately so far (i.e. monitored at least on an hourly basis over several hours, at high signal-tonoise $\mathrm{S} / \mathrm{N}$ and high spectral resolution) show stochastically varying, narrow emission subpeaks superposed on broad emission lines. This is the case for some ten WR stars (Robert 1992; Lépine 1998; Lépine \& Moffat 1999), two [WC]-type central stars of planetary nebulae (Balick et al. 1994; Grosdidier et al. 1997), one Of star (Eversberg et al. 1998) and at least one nova (Garnavitch, priv. comm.). It is strongly suspected that this is actually a very widespread phenomenon, probably present in all hot-star winds. The most likely interpretation is that we are seeing the manifestation of a hierarchy of density enhancements, often referred to as "clumps", "blobs" or "shocks", propagating outwardly with the wind. In fact, they probably are the wind.

2. Recurrent (R). Broad subpeaks are seen in only a small number of WR winds (Morel 1998; Morel et al. 1998a, 1998b). They tend move across the whole emission line and recur, but only over several cycles. Among the 30 or so WR stars which are apparently bright enough to have been checked for this kind of LPV, only WR6 and WR134 appear to fall in this category, with the possible addition of WR1 (Morel, priv. comm.). These stars also appear to exhibit S-type LPVs, which are often masked by those of R-type. WR11 $=\gamma^{2}$ Vel also shows broad variable subpeaks, but these apparently behave in a stochastic fashion and belong to the $S$ category (Lépine et al. 1998). It is quite noteworthy that WR6 and WR134 are the single WR stars showing the largest amount of line depolarisation (Schulte-Ladbeck 1994; Harries et al. 1998). WR1 is not exceptional at all in this way, however, and another WR star with moderately srong line depolarisation is the WR component in the long-period binary WR137.

The current interpretation of the $\mathrm{R}$ scenario in WR winds is that we are witnessing the rotational modulation of a persistant large-scale anisotropic outflow, which is likely related to the propagation of discrete absorption components (DACs) and corotating interaction regions (CIRs) in OB-star winds (e.g. Cranmer \& Owocki 1996). While these are seen frequently in $\mathrm{OB}$ winds, they are rare in WR winds. Presumably they are generated by magnetic structures in forced rotation or non-radial pulsations close to the stellar (hydrostatic) surface, the former being somewhat more likely. Such features do not last more than several rotations, hence are incoherent on long timescales.

3. Periodic (P). Only binary systems appear to reveal truly periodic LPVs in WR and other hot stars. These are normally interpreted as arising in cyclic modulations due to wind-wind collisions, photospheric and atmospheric eclipses, heating effects and other binary-related distortions (see Marchenko et al. 1997). 


\section{Evidence for Stochastic Line Profile Variations}

In this review, I will limit further discussion to the S type LPVs. As noted above, these small-scale, stochastic variations are probably omnipresent in all WR and other weaker hot-star winds. We now look in more detail at the evidence for these stochastic line profile variations in WR winds. To do this, it is useful to divide up the evidence into classes according to the degree of sophistication of the observations, reflected by their "dimension":

0-D. Here the manifestation of the variations are characterised by a quantity which gives no information about the detailed nature of the actual 3-D clumping: it could be e.g. shells or clumps. In order of $\sim$ decreasing wavelength, there are a large number of 0-D signatures of clumping:

- IR/radio excess, compared to the thermal emission from the underlying star (Runacres \& Blomme 1996; Blomme \& Runacres 1997). The level of the excess can only be accounted for if clumping occurs, since the f-f emission excess goes as the square of the density.

- Increase of the eclipse width and depth especially at secondary minimum (O-star in front) of the IR compared to the optical light curve of the WR + O eclipsing system V444 Cyg (Cherepashchuk et al. 1984). Again, this must be due to the enhanced f-f emission due to some kind of clumping of the WR wind, although it provides no indication of the true nature of the clumping.

- Dust formation in WC star winds. In such a hostile environment, localised density enhancements are needed both to form the dust and once formed, to shield it from the intense UV radiation field of the central star (Cherchneff et al. 1998). This can occur either in the winds of single, cool WC stars (most WC9-10 and some WC8 stars) or in the wind-wind collision zone in some WC + O binaries (Williams 1996), both leading to IR excess. Additional evidence for dust formation comes from episodic dips in the continuum light curves of some WC9 stars (Veen et al. 1998). Again, no real constraints are provided as to the true nature of the clumping.

- Photometric (e.g. Moffat \& Shara 1986) and polarimetric (Robert et al. 1989) continuum variability. The stochastic nature of this variability on timescales of hours to days suggests some kind of clumping in the winds. The fact that the polarisation scatter is normally much less than the photometric scatter implies that the clumps both scatter and emit light (Brown et al. 1995).

- Distortion of line intensity ratios. Nugis \& Niedzielski (1995) find an enhancement of the flux ratio of HeI to HeII lines in the IR, that allows one to deduce that clumping must be important.

- Disk-shaped HeI line profiles. These can be reproduced if clumping is important (Antokhin et al. 1992).

- Diffuse electron scattering (Hillier 1991). This creates an enhancement on the red side of emission line profiles, due to scattering in an expanding 
medium around the star. Since (optically thin) electron scattering is independent of clumping, this enhanced component is correspondingly less than the main direct emission component for the same line when the wind is clumped than when it is smooth.

- Saturation of P Cygni absorbtion profiles (Prinja et al. 1990). This is a consequence of a non-monotonic velocity expansion law of the wind, caused by velocity scatter due to shocks in the wind; a dispersion of $\sim 10^{2} \mathrm{~km} / \mathrm{s}$ is implied.

- High X-ray fluxes from hot-star winds (e.g. Berghöfer \& Schmitt 1994). Although current X-ray satellites are not large enough yet to detect the expected stochastic X-ray variability, the observed X-ray fluxes imply that shocks must be present in the wind, with thermal temperatures of $\sim 10^{6}$ K (Wesselowski 1996; Feldmeier et al. 1997).

- Erratic X-ray variability in massive X-ray binaries (Kaper et al. 1996). This could be caused either by clumped winds being accreted onto the compact companion, or by instabilities within the accretion disk (if there is one).

1-D. Doppler imaging has now enabled us to separate out various clumps in the wind and at least partly qualify the nature of the clumping. Only one observational signature so far falls in this category for the otherwise unresolved inner WR wind:

- LPV. The information content is greater for emission lines, which arise globally in the wind, compared to $\mathrm{P}$ Cygni absorptions, which probe the highly localised column of the wind from the observer to the star. The latter is somewhat ambiguous, unable to clearly distinguish between DACs and clumps. In any case, most WR resonance P Cygni absorptions in WR stars are saturated, making their study virtually impossible except at their sloping edges. Global emission line variability has been detected by various groups now (e.g. Schumann \& Seggewiss 1975; Moffat et al. 1988; McCandliss 1988; Robert 1992; Lépine 1998); an update of previous results is given in the next section.

2-D. Resolution of the outer parts of WR winds has now been accomplished via direct imaging in two special cases. Note that even the closest WR star, $\gamma^{2}$ Vel, would require a resolution of $\sim 2$ mas to distinguish $1 \mathrm{R}_{\odot}$, a possible clump size in the inner wind, at its (new) distance of $\sim 250 \mathrm{pc}$.

- Radio interferometry. The second closest WR star, WR147 (d 700 pc), has been resolved using MERLIN into a thermally clumped wind on a scale of $\sim 20$ mas $\left(10^{3} R_{\star}\right)$ (Williams et al. 1997). We (Barlow et al.) hope to do the same soon for $\gamma^{2}$ Vel.

- Optical line imaging of the young WR nebula M1-67 around WR124. Grosdidier et al. (1998) have used HST/WFPC2 to resolve this nebula in $\mathrm{H} \alpha$, which is filled in right down to the observable limit close to the central star. This remarkable image shows, among other interesting features, a number of 
strange point-like sources of bright $\mathrm{H} \alpha$ emission surrounded by what appear to be their own local wind blown bubbles. Lack of bow-shock shapes implies that they are travelling out with the ambient wind, suggesting that we might be seeing a few of the largest wind clumps emerging from a previous (slower) LBV stage. The alternative speculation that we are seeing some new kind of instability in the wind lacks a theoretical understanding. Some of these ideas may change, as Grosdidier et al. (1999) are in the process of examining the velocity field across M1-67, based on new high-resolution Fabry-Pérot observations in $\mathrm{H} \alpha$ from CFHT.

3+ -D. Increased resolution might be provided by $2-\mathrm{D}$ imaging combined with velocity (RVs or pm's) or other information. One can only dream for now of Vakili's (this conference) technique of "interfero-spectropolarimetry"!

\section{Highlights of New Work on S-type LPVs}

\subsection{Data Source and Technique}

Based on the same spectral data reported in Robert (1992), Lépine et al. (1996) and Lépine \& Moffat (1999) have significantly improved the analysis of the $9 \mathrm{WR}$ stars for line variability. These data comprise 3-4 contiguous nights of $\mathrm{R} \sim 30000, \mathrm{~S} / \mathrm{N} \gtrsim 200 /$ pixel (continuum), $\delta \mathrm{t} \sim 1$ h spectrosocpy of the best optical line in a range of WN and WC subtypes.

The key here is twofold: (1) Wavelet analysis has been applied to the line variations relative to a mean profile. This is a better alternative to Fourier techniques or temporal variance spectrum analyses (Fullerton 1990): wavelets allow one to analyse the pattern as a whole, rather than trying to extract individual clumps or looking at one pixel at a time. (2) A phenomenological model has been devised to simulate the observed LPVs. The model is calculated with the same sampling as the observations, in velocity space using a $\beta$ velocity law to relate projected acceleration to velocity. The model also allows for a scaling power law for the fluxes of the clumps (although not in velocity dispersion) as expected if turbulent cascading is acting. From these simulations, it was deduced that superposition effects are generally very important, leading to a high level of confusion and change in interpretation compared to previous studies (e.g. Moffat et al. 1994).

\subsection{Results}

One of the WR stars (WR134) in the Robert (1992) sample is dominated by R-type LPVs (cf. Morel et al. 1998b for a more detailed study of this star). The remaining 8 stars all show very similar S-type LPV patterns, which can be summarized as follows:

- All the most obvious emission subpeaks in all stars have velocity widths of FWHM $\sim 2-10 \AA$ and amplitudes of $\sim 2-8 \%$ of the average line intensity. 
- Subpeak motion occurs always away from line centre, both in the blue and red directions. This can be most readily interpreted as clumps propagating radially outward in a spherically symmetric wind, and seen in velocity projection $\mathrm{v}(\mathrm{r}) \cos \theta$, each with $\theta \sim$ const.

- Each observed subpeak generally is the sum of a large number of independent, discrete wind emission elements (DWEEs). These are assumed to be Gaussian in shape in the simulations.

- A large number of DWEEs $\left(z 10^{4}\right)$ are needed to account for the low degree of variability observed. This implies that DWEEs must occupy a small volume.

- DWEEs reveal a large velocity dispersion, with dominant scale $\sigma_{v} \sim$ $100 \mathrm{~km} / \mathrm{s}(\sim 350 \mathrm{~km} / \mathrm{s}$ in WR134). This is likely a consequence of macroturbulence and associated shocks.

- A strong anisotropy (radial versus tangential) prevails in velocity dispersion within the DWEEs: $\sigma_{v_{r}} \sim 4 \sigma_{v_{t}}$. This is compatible with radiative wind driving (e.g. Rybicki et al. 1990). For WR134 $\sigma_{v_{r}} \sim \sigma_{v_{t}}$, implying significant angular motion, as expected for R-type LPV.

- There is marginal evidence for optical depth effects within the DWEEs, implying that the escape probablility for photons may be slightly less in the radial than in the transverse direction.

- Relatively low radial acceleration is found in the inner wind and high acceleration in the outer wind, characterised by the parameter product $\beta R_{\star} / R_{\odot}$ $=20 \ldots 80$, much larger than obtained from most "standard model" fits, with $\beta \sim 1$ and $R_{\star}$ of several $R_{\odot}$.

- The duration of subpeak events agrees with the overall line shape, implying a relatively thin line-emitting region (LER) in velocity space.

- The stochastic behaviour of the DWEEs agrees with model simulations (e.g. Gayley \& Owocki 1995), with rapid growth of random fluctuations near the base out to the observed wind.

- The same phenomenon appears to be at work in all WR winds, independent of the spectrum and subclass, e.g. $\sigma_{v}$ is independent of the geometry of the LER, $v_{\infty}$, wind acceleration, ...

However, there still remains one important factor, which turns out to be a rather severe limitation: The number of visible subpeaks in any given line in a given star is proportional to $v_{\infty}$, as noted by Robert (1992). Does this mean that faster winds have more structure? The answer now appears to be $N O$ ! Rather, it is spurious, due to superposition effects that are worst in narrow-line (slower-wind) stars. Their is no way around this problem, although observing stars with large $v_{\infty}$ does help somewhat. Higher spectral resolution or $\mathrm{S} / \mathrm{N}$, or shorter $\delta \mathrm{t}$ will not help much. 


\section{Importance of Clumping}

From studies of subpeak behaviour on WR emission lines, the following conclusions can be stated regarding the associated wind clumping:

- Clumping is probably universal in hot-star winds. There is no reason why WR stars should be special, and in any case, evidence exists already for clumping in other types of hot stars.

- All previous smooth-wind based mass-loss rates will have to be reduced by about a factor three. This comes from both observation (polarization modulation and period changes in WR binaries: St-Louis et al. 1993; Moffat \& Robert 1994) and theory (e.g. Schmutz 1997; Hillier \& Miller 1998; Nugis et al. 1998).

- The revision in mass-loss rates will have an important impact on models of stellar evolution.

- Clumping is probably crucial to explain the formation of dust in WC winds.

- WR winds appear to be excellent laboratories to study turbulence in action.

- Propagating inhomogeneities allow us to trace the wind kinematics, e.g. derive a relation between the observables acceleration and velocity, and thence deduce the velocity law $\mathrm{v}(\mathrm{r})$.

- Clumping will tend to "soften" collisions, e.g. with the ISM or other winds.

- Clumpy winds will make a direct contribution to the clumpiness of the ISM.

\section{$5 \quad$ Future Prospects}

Several avenues remain to be exploited:

- Resolving clumps interferometrically. This has already started in the radio and will soon (in 10 years from space?) be possible in the optical/IR. Some current "dirty laudry" is likely to take a severe cleaning...

- Spectroscopy: larger samples of stars of different type, longer continuous time-coverage and probing different lines simultaneously.

- Exploring internal structure of clumps, other scaling laws, and establishing a clear relation to turbulence.

- More modeling is necessary: 3-D hydrodynamics with radiative effects included and more quantitative comparisons with observations; calculating CIRs/DACs in WR winds.

\section{References}

Antokhin, I.I., Nugis, T., Cherepashchuk, A.M. (1992): Sov. Astron., 36, 260

Balick, B., et al. (1996): AJ, 111, 834 
Berghöfer, T.W., Schmitt, J.H.M.M. (1994): ApSpSc, 221, 309

Blomme, R., Runacres, M.C. (1997): A\&A, 323, 886

Brown, J.C., et al. (1995): A\&A, 295, 725

Cherchneff, I., et al. (1998): A\&A, submitted

Cherepashchuk, A.M., Khaliullin, K.E., Eaton, J.A. (1984): ApJ, 281, 774

Cranmer, S.R., Owocki, S.P. (1996): ApJ, 462, 469

Eversberg, T., Lépine, S., Moffat, A.F.J. (1998): ApJ, 494, 799

Feldmeier, A., Puls, J., Pauldrach, A.W.A. (1997): A\&A, 322, 878

Fullerton, A.W. (1990): Ph.D. Thesis, Univ. of Toronto

Gayley, K.G., Owocki, S.P. (1995): ApJ, 446, 296

Grosdidier, Y. et al. (1997): IAU Symp. No. 180, 108

Grosdidier, Y., et al. (1998): ApJ, 506, L127

Grosdidier, Y., et al. (1999): in preparation

Harries, T.J., Hillier, D.J., Howarth, I.D. (1998): MNRAS, 296, 1072

Hillier, D.J. (1991): A\&A, 247, 455

Hillier, D.J., Miller, D.L. (1998): ApJ, 496, 407

Kaper, L., et al. (1996): A\&AS, 116, 257

Lépine, S. (1998): Ph.D. Thesis, Univ. de Montréal

Lépine, S., Moffat, A.F.J., Henriksen, R.N. (1996): ApJ, 466, 392

Lépine, S., Eversberg, T., Moffat, A.F.J. (1998): ApJ, submitted

Lépine, S., Moffat, A.F.J. (1999): ApJ, 510 (1 Jan)

Marchenko, S.V., et al. (1997): ApJ, 485, 826

McCandliss, S.R. (1988): Ph.D. Thesis, Univ. of Colorado, Boulder

Moffat, A.F.J., et al. (1988): ApJ, 334, 1038

Moffat, A.F.J., Shara, M.M. (1986): AJ, 92, 952

Moffat, A.F.J., Robert, C. (1994): ApJ, 421, 310

Moffat, A.F.J., et al. (1994): ApSpSc, 216, 55

Morel, T. (1998): Ph.D. Thesis, Univ. de Montréal

Morel, T., et al. (1998a): ApJ, 498, 413

Morel, T., et al. (1998b): ApJ, in press

Nugis, T., Niedzielski, A. (1995): A\&A, 300, 237

Nugis, T., Crowther, P.A., Willis, A.J. (1998): A\&A, in press

Prinja, R.K., Barlow, M.J., Howarth, I.D. (1990): ApJ, 361, 607

Robert, C., et al. (1989): ApJ, 347, 1034

Robert, C. (1992): Ph.D. Thesis, Univ. de Montréal

Runacres, M.C., Blomme, R. (1996): A\&A, 309, 544

Rybicki, G.B., Owocki, S.P., Castor, J.I. (1990): ApJ, 349, 274

Schmutz, W. (1997): A\&A, 321, 268

Schulte-Ladbeck, R. (1994): ApSpSc, 221, 347

Schumann, J.D., Seggewiss, W. (1975): IAU Symp. No. 107, 299

St-Louis, N. (1990): Ph.D. Thesis, Univ. College London

St-Louis, N., et al. (1993): ApJ, 410, 342

Veen, P., et al. (1998): A\&A, 329, 199

Wesselowski, U. (1996): 33rd Liège Ap Coll, 343

Williams, P.M. (1996): 33rd Liège Ap. Coll., 135

Williams, P.M., et al. (1997): MNRAS, 289, 10 


\section{Discussion}

G. Koenigsberger: The radio emission associated with the WR 147 windwind collision region seems very broad. Could you comment on this?

A. Moffat: According to wind-wind collision models (e.g., Stevens et al. 1992), the relative separation of the shock fronts on either side of the contact surface can indeed be quite large, especially if the collision is more or less adiabatic. This is likely to be the case in long-period systems like WR 147.

A. Feldmeier: Do you think that there is a connection between the X-ray emission from OB stars and the strongest blobs you find? Say, if the blob gas is the cool, dense gas behind the strong X-ray emitting reverse shock, then (since the radiative cooling zone is isobaric) $\rho \mathrm{T}=$ const and one could expect that the largest blob overdensities are given by $\rho_{\text {blob }} / \rho_{\mathrm{CAK}} \sim \mathrm{T}_{\mathrm{X}-\text { ray }} / \mathrm{T}_{\text {wind }} \sim$ 100. Can you derive the largest overdensities from your observations?

A. Moffat: Unfortunately, with the extremely large number of estimated clumps $\left(>10^{4}\right)$ combined with the ambiguities (superposition) of spectroscopy, we cannot provide any serious constraints on the density contrast. If compressible turbulence is at play, the small (less massive) clumps are expected to be densest, so that the $\mathrm{X}$-rays could arise in a very large number of clumps.

A. Maeder: Does the revision of the mass-loss rates you are suggesting (factor of $\sim 3$ ) also apply to O-type and WR stars?

A. Moffat: We only have real line-profile variability data for the extreme $O$ star $\zeta$ Pup. In that case, the stochastic variability is every bit as high as any WR star in the same line relative to the average line emission. We can only guess that this might lead to similar corrections for $\zeta$ Pup. It would not surprise me if all hot-star winds were clumpy, but more work is needed to show what the impact of that might be.

H. Lamers: The mass loss rates from $O$ stars are usually derived from $P$ Cygni profiles.

S. Shore: In the binary WR + O system, is it possible that you would see a change in the stochastic variations of the emission lines with the binary phase? For instance, in V 444 Cyg there is a hole in the wind from the OWR wind collision, so you might expect to see different wavelet spectra as a function of time.

A. Moffat: In V 444 Cyg, most of the variations are phase dependent, which masks the stochastic component to a large extent. In $\gamma$ Vel, we find no significant phase dependence of the stochastic line variation component around more or less half the orbit. 